\title{
Síndrome de Goldenhar: relato de caso
}

\author{
Jener G. Farias ${ }^{1}$ \\ Christiano Oliveira ${ }^{2}$ \\ Hamurabi B. Santiago ${ }^{3}$ \\ Paulo S. F. Campos' \\ Gleicy G. V. S. Carneiro5 \\ Mayle M. Meirelles
}

\begin{abstract}
Resumo
A Síndrome de Goldenhar é uma condição rara, geralmente de ocorrência esporádica, caracterizada por microssomia hemifacial, dermóide epibulbar, más formações do pavilhão auditivo e anomalias vertebrais. Existem também relatos de associações da síndrome com outras condiçōes sistêmicas. Os autores relatam um caso de Síndrome de Goldenhar em paciente do sexo feminino, 28 anos, sem antecedentes familiares, que apresentava microssomia hemifacial, o que comprometia os músculos da mastigação, a expressão facial e os ossos da face (mandíbula, maxila e zigomático) do lado esquerdo, presença de broto auricular, história médica de dermóide epibulbar e fissura lateral de face, ipsilateralmente. $\mathrm{Na}$ avaliação sistêmica, foram encontrados ectopia do rim esquerdo e discreto espessamento dos folhetos mitrais, com refluxo mitral de grau leve.
\end{abstract}

Palavras-chave: Síndrome de Goldenhar; displasia óculo-aurículo-vertebral; defeitos congênitos.

\section{INTRODUÇÃO}

A síndrome de Goldenhar (SG) foi primeiramente descrita em 1952 por Maurice Goldenhar como um conjunto de anomalias congênitas do primeiro e segundo arcos branquiais, que incluem dermóide epibulbar, apêndices auriculares e más-formações do pavilhão auditivo. Gorlin, mais tarde, acrescentou as anomalias vertebrais a essa entidade clínica e sugeriu o termo displasia óculo-aurículo-vertebral $(1,2)$.
Também chamada de síndrome do primeiro e segundo arcos branquiais e displasia facial lateral, a síndrome de Goldenhar apresenta como sinais clínicos anormalidades auriculares (protuberâncias ou fístulas préauriculares, má formação do ouvido médio ou interno, com perda auditiva), anormalidades oculares (dermóides ou lipodermóides epibulbares, coloboma de pálpebra superior, dermolipoma subconjuntival ou orbital anteri-

\footnotetext{
${ }^{1}$ Mestre em Cirurgia e Traumatologia buco-maxilo-facial (CTBMF) pela Faculdade de Odontologia da Universidade de Pernambuco. Recife - PE

${ }^{2}$ Bolsista de Iniciação Cientifica da FAPESB. Faculdade de Odontologia da UFBA. Salvador - BA

${ }^{3}$ Médico especialista em Saúde da Família - FBDC. Salvador - BA

${ }^{4}$ Prof. Adjunto de Radiologia da Faculdade de Odontologia da UFBA. Salvador - BA.

${ }^{5}$ Cirurgiã-dentista do Serviço de Estomatologia e Cirurgia Buco-maxilo-facial do Hospital Santa Casa de São Felix. São Felix - BA.

Correspondência para / Correspondence to:

Christiano Oliveira

Rua Rodrigo Argolo, 57/403, Rio Vermelho.

41.940-220. Salvador - Bahia - Brasil.

Tel.: (71) 3240-2178 / Fax. (71) 3341-8348.

E-mail: oliveirachristiano@hotmail.com.
} 
or) e alterações vertebrais (espinha bífida, occiptalização do atlas, fusão de vértebras, escoliose cérvico-torácica). As anormalidades buco-maxilo-faciais incluem hipoplasia do ramo mandibular, assimetria facial, fissuras faciais laterais, hipoplasia dos ossos zigomático, temporal e maxilar, dos músculos da mastigação e da glândula parótida $(3,4)$. Relatos têm sido feitos sobre a associação da Síndrome de Goldenhar com outras condições como alterações genitourinárias $(3,5)$, cardiovasculares $(6,7)$, desordens psiquiátricas $(8,9)$, apnéia obstrutiva do sono (10).

A SG é uma condição rara, cuja etiopatogenia multifatorial está relacionada a anormalidades vasculares ou hemorragias na região do primeiro e segundo arcos branquiais, que resultam em distúrbios no desenvolvimento embriológico, sendo geralmente de ocorrência esporádica, embora casos familiares já tenham sido relatados $(2,11)$. A heterogeneidade dos fatores etiológicos e ainda o estágio de desenvolvimento em que o embrião é acometido determinam um amplo espectro de manifestações da síndrome. A carência de critérios e a variação no grau de acometimento são fatores que dificultam o diagnóstico e o acompanhamento dos pacientes.

O diagnóstico da SG não deve basear-se apenas em achados radiográficos ou laboratoriais, devendo resultar de exames de anormalidades sistêmicas associadas, incluindo avaliação auditiva, genito-urinária (3), vertebral, SNC, ecocardiograma, exame polissonográfico $(3,10)$. A Síndrome de Treacher-Collins, arrolada no diagnóstico diferencial, geralmente tem acometimento bilateral e está associada a herança autossômica dominante (2), o que não é evidenciado na SG.

$\mathrm{O}$ tratamento instituído deve ser individualmente determinado (12). Para um acompanhamento adequado, é recomendada uma abordagem multidisciplinar, buscando melhorar a aparência e a funcionalidade das estruturas acometidas. Uma intervenção precoce contribui para a promoção de um desenvolvimento normal do indivíduo. Recomenda-se a remoção cirúrgica do dermóide epibulbar, em função de distúrbios visuais, ou por necessidade estética. Para a promoção de um correto desenvolvimento da fala, recomenda-se correção cirúrgica e (ou) utilização de prótese, com posterior acompanhamento fonoaudiológico (1).

A distração osteogênica tem sido utilizada na correção da assimetria facial e consiste em um método de formação óssea através de osteotomia e afastamento seqüencial, com utilização de aparelhagem específica, trazendo resultados estéticos aceitáveis e correção do plano oclusal para uma condição funcional (13).

\section{RELATO DO CASO}

Paciente do sexo feminino, 28 anos, com alterações morfológicas em face, foi encaminhada ao serviço de Estomatologia e cirurgia e traumatologia buco-maxilo-facial da Santa Casa de São Félix-BA. Apresentava microssomia hemifacial à esquerda, com hipoplasia dos músculos da mastigação e expressão facial e dos ossos da face (mandíbula, maxila e zigomático) (FIGURA 1 a 3). Incluem-se, nas alteraçôes faciais, broto auricular, mancha tipo névoa em região lateral do olho esquerdo, secundário à exérese de um dermóide epibulbar, além de uma fissura lateral, corrigida cirúrgica e previamente à admissão, que se estendia horizontalmente da comissura labial até o pavilhão auricular externo, do mesmo lado esquerdo (FIGURA 4).

Não havia registro de condições pré-natais nem história familiar positiva para casos similares. Exames complementares evidenciaram alterações morfológicas mandibulares no processo coronóide e côndilo esquerdos, hipoplasia da região, com redução da espessura do ramo ascendente e irregularidade na implantação dos dentes, bem como calcificação bilateral do ligamento estilo-hióideo. Foi também observada uma lordose lombar fisiológica (postural), e não foram encontradas alterações da densidade, estrutura, dimensões ou formas das vértebras. A ultrassonografia do aparelho urinário revelou ectopia (pélvica) do rim esquerdo e desvio do rim direito para a linha média. $\mathrm{O}$ ecocardiograma com dopplerfluxometria demonstrou discreto espessamento dos folhetos mitrais, com refluxo mitral de grau leve. Não foram encontradas alterações laboratoriais dignas de nota. 


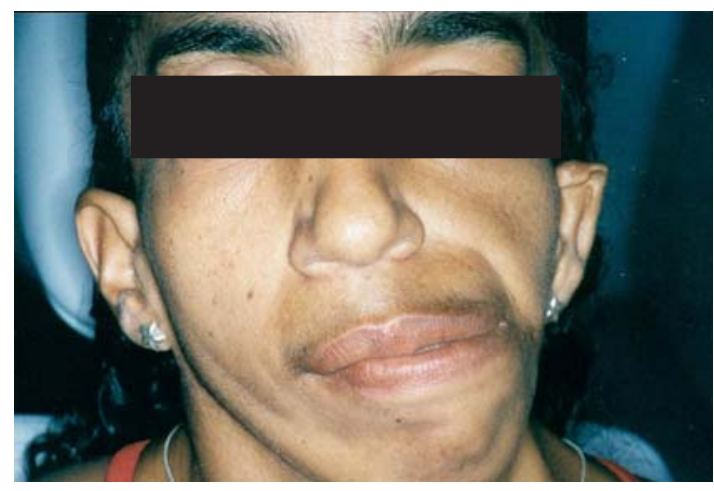

Figura 1 - Fotografia frontal evidenciando a microssomia hemifacial no lado esquerdo, com severo desvio da linha média.

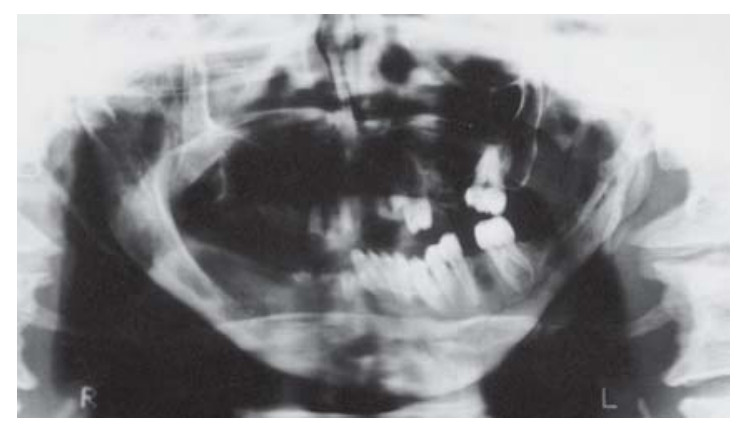

Figura 3 - Radiografia panarômica confirmando a microssomia hemifacial esquerda.

\section{DISCUSSÃO}

Ishytoia e colaboradores (3) afirmam que os portadores da SG podem apresentar graus variados de alguns ou todos os sinais relatados. Essa observação pôde ser constatada nesse caso clínico. A paciente apresenta sinais clássicos da síndrome como microssomia hemifacial com atrofia dos músculos da mastigação e expressão facial e ossos da face, dermóide epibulbar, brotos auriculares e má-formação do aparelho auditivo, levando a disacusia no lado esquerdo. Scholtz e colaboradores (12), em um estudo histopatológico post-mortem do osso temporal em um caso de SG, associaram a surdez a uma deficiência condutiva, descartando a presença de componentes neurossensoriais relacionados. A fissura lateral da face parece ser um achado menos comum. Não foram encontradas alterações vertebrais, como descritas em vários casos

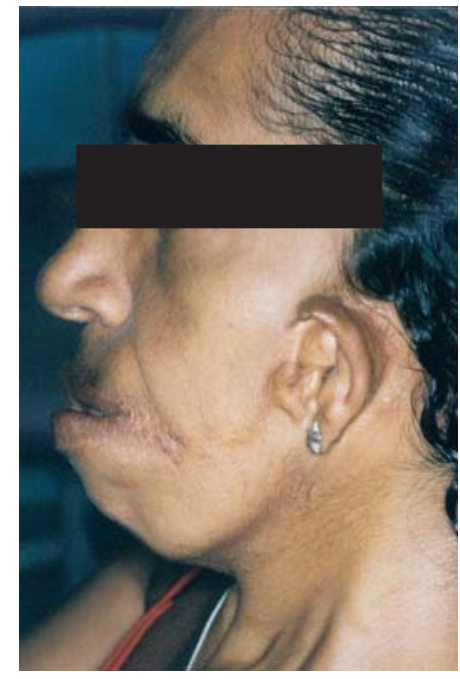

Figura 2 - Fotografia lateral demonstrando fissura facial corrigida cirurgicamente e presença de brotos auriculares

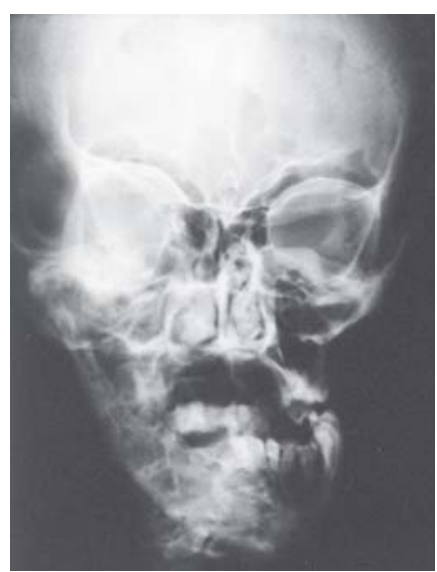

Figura 4 - Fotografia lateral demonstrando fissura facial corrigida cirurgicamente e presença de brotos auriculares.

na literatura (3), demonstrando a ocorrência de forma incompleta, no caso em questão.

A paciente negou história de quadros similares na família, sugerindo ocorrência esporádica da doença. Não há ainda uma etiologia definida para essa síndrome. Encontra-se, na literatura, sua associação com uma trissomia no cromossomo 9, ou ainda distúrbios nas células da crista neural (14). O uso materno, durante a gestação, de drogas com conhecido potencial teratogênico, como talidomida, ácido retinóico, 
primidona, tamoxifeno e cocaína, também tem sido relacionado ao desenvolvimento dessa patologia $(9,15)$. Pinheiro e colaboradores (2) sugerem ainda a participação dos anticonvulsivantes fenobarbital e fenitoína, na sua etiopatogenia. É também sugerida a sua associação com a diabetes materna (4). Na história médica materna, porém, não constavam alterações no período da gestação. Foram negadas intercorrências gestacionais ou uso de drogas durante a gravidez.

Anomalias em outros órgãos têm sido relatadas. Alterações cardiovasculares têm sido descritas em 5 a $58 \%$ dos pacientes portadores dessa síndrome $(6,7)$. O prognóstico dessa condição depende, freqüentemente, das condiçōes cardiovasculares subclínicas. O espectro de alterações desse sistema varia até severos distúrbios congênitos, como tetralogia de Fallot e dextrocardia (6). No caso relatado, foi encontrado um leve espessamento dos folhetos mitrais, levando ao refluxo, mas sem alterações funcionais relevantes.

A incidência de associação entre más-formações genito-urinárias e a SG ainda não está bem definida. Todavia, anormalidades renais e ureterais foram descritas em até $70 \%$ dos pacientes (5), podendo acarretar obstrução do trato urinário, com posterior dilatação pielocalicial e insuficiência renal. Não foram observadas alterações funcionais desse sistema na paciente em estudo, apesar de haver sido visualizada ectopia renal à esquerda e desvio do rim direito. Ritchey e colaboradores (5) encontraram ectopia renal em $40 \%$ dos pacientes.

O tratamento instituído para a síndrome de Goldenhar deve ser individualmente determinado e com abordagem multidisciplinar. No presente relato, para a adequada abordagem do caso, faz-se necessária a atuação dos serviços de cirurgia buco-maxilo-facial, cirurgia plástica, reabilitação oral, além do acompanhamento clínico e fonoaudiológico. A reconstrução dos ossos gnáticos pode ser alcançada pela expansão da mandíbula através de enxerto ósseo ou da técnica de distração osteogênica (13). Essa última consiste na utilização de uma aparelhagem específica, com a finalidade de produzir expansão óssea progressiva, através do afastamento de segmentos ósseos, após osteotomia. Essa técnica possui como vantagens o nivelamento do plano oclusal, além de requerer apenas um sítio cirúrgico, em oposição à enxertia, que requer dois sítios cirúrgicos e está associada a maior morbidade (13). Ademais, a distração possibilita uma melhor acomodação e expansão também dos tecidos moles; e o osso neoformado possui o mesmo diâmetro e resistência do osso adjacente.

As adequações estéticas demandadas pela paciente, como a exérese do apêndice préauricular e a remoção da cicatriz deixada pela correção da fissura facial, podem ser alcançadas por técnicas de cirurgia plástica. $\mathrm{O}$ tratamento ortodôntico tem sido indicado em casos de máposição dentária associada à microssomia hemifacial e deve ser instituído precocemente, para a obtenção de melhores resultados. No caso relatado, verificam-se ausências de unidades dentárias hígidas (FIGURA 3), sendo indicado, portanto, tratamento de reabilitação oral funcional e estética, através de exodontias dos restos radiculares, com posterior confecção de próteses ou implantes dentários. O acompanhamento fonoaudiológico auxiliaria a paciente na re-educação fonética. E as anomalias cardiovasculares e renais encontradas, não obstante não terem demonstrado repercussões clínicas até o presente, não devem ser negligenciadas.

\section{COMENTÁRIOS}

Não existe um consenso na literatura quanto à terminologia adequada para se referir à condição. Enquanto alguns artigos se referem à microssomia hemifacial como sinônimo de SG, outros tratam esse termo como uma das alterações componentes da síndrome. Concordamos com a maioria dos autores, considerando como sinônimos os termos Síndrome de Goldenhar e Espectro Óculo-aurículo-vertebral, e entendemos que a microssomia hemifacial faz parte do quadro clínico da SG.

No caso apresentado, não foram diagnosticadas alterações vertebrais, tratandose da forma incompleta da síndrome, que apresenta, como já visto, um amplo espectro de ma- 
nifestaçōes. A paciente foi encaminhada ao Serviço de Genética da Universidade Federal da Bahia e atualmente se encontra sob os cuidados da equipe do Centro de Deformidades da Face do Hospital Santo Antônio, em Salvador, em fase de planejamento e exames pré-cirúrgicos.
Este relato de caso objetiva auxiliar os profissionais de saúde no adequado reconhecimento e abordagem dos pacientes, permitindo uma atuação precoce, o que proporcionaria um desenvolvimento adequado da face e maior qualidade de vida aos portadores dessa síndrome.

\title{
Goldenhar's syndrome: a case report
}

\begin{abstract}
Goldenhar's syndrome is a rare condition, usually sporadic, characterized by hemifacial microsomia, epibulbar dermoid, malformations of the external ear and vertebral anomalies. Associations with systemic conditions have been reported. The authors report one case of Goldenhar's syndrome in a 28-year-old female, without familial occurrence reported, presenting hemifacial microsomia affecting mastication and facial expression muscles and facial bones (mandible, maxilla, and zygomatic) on the left side, ipsilateral preauricular tags, and medical history of epibulbar dermoid and lateral facial cleft. Systemic features included ectopic left kidney, slight thickening of mitral valve and mild mitral regurgitation.
\end{abstract}

Keywords: Goldenhar's syndrome; oculoauriculovertebral dysplasia; birth defects.

\section{REFERÊNCIAS}

1 MOCELLIN, M. et al. Síndrome de Goldenhar (Displasia oculoauriculovertebral): relato de caso e revisão de literatura. R. Bras. Otorinolaringol., Porto Alegre, v.64, n.1, p.7779, 1998.

2 PINHEIRO, A.L.B. et al. Goldenhar's syndrome: case report. Braz. Dent. J., Ribeirão Preto, v.14, n.1, p.67-70, 2003.

3 ISHITOYA, S. et al. Left retrocaval ureter associated with the Goldenhar Syndrome (Branchial Arch Syndrome). J. Urol., Hagerstown, v.158, n.2, p.572-573, 1997.

4 WANG, R.; MARTINES-FRIAS, M.L.; GRAHAM, J.M. Infants of diabetic mothers are at increased risk for the oculo-auriculovertebral sequence: a case-based and case-control approach. J. Pediatr., St. Louis, v.141, n.5, p.611-621, 2002.

5 RITCHEY, M.L. et al. Urologic manifestations of Goldenhar syndrome. Urology, New York, v.43, n.1, p.88-91, 1994.
6 KUMAR, A. et al. Pattern of cardiac malformation in oculoauriculovertebral spectrum. Am. J. Med. Genet., New York, v.46, n.4, p.423-426, 1993.

7 NAKAJIMA, H. et al. Goldenhar syndrome associated with various cardiovascular malformations. Jpn. Circ. J., Kyoto, v.62, n.8, p.617-620, 1998.

8 BRIEGAR, P. et al. Oculo-auriculo-vertebral disorder (Goldenhar "syndrome») coexisting with schizophreniform disorder. J. Neurol. Neurosurg. Psychiatr., London, v.65, n.1, p.135-136, 1998.

9 LLEONART, M.B. et al. Displasia oculoauriculovertebral o Síndrome de Goldenhar: estudio multidisciplinario de un caso clínico. R. Cubana Oftalmol., La Habana, v.14, n.1, p.42-46, 2001.

$10 \mathrm{HOCH}, \mathrm{B}$; HOCHBAN, W. Four-yearold girl with Goldenhar-sequence and severe obstructive sleep apnea, symptoms, diagnosis 
and therapy. Int. J. Pediatr. Otorhinolaryngol., Limerick, v.43, p.277-281, 1998.

11 CHOONG, Y.F. et al. Goldenhar and Cri$\mathrm{du}$-Chat syndromes: a contiguous gene deletion syndrome? J. AAPOS, St. Louis, v.7, n.3, p.226-227, 2003.

12 SCHOLTZ, A.W. et al. Goldenhar's syndrome: congenital hearing deficit of conductive or sensorineural origin? Temporal bone histopathologic study. Otol. Neurotol., Hagerstown, v.22, n.4, p.501-505, 2001.

13 WIENS, J.L.; FORTE, R.A.; WIENS, J.P. The use of distraction osteogenesis to treat hemifacial microsomia: a clinical report. J. Prosthet. Dent., St. Louis, v.89, p.11-14, 2003. 14 WILSON, B.N.; BARR JR, M. Trisomy 9 mosaicism: another etiology for the manifestations of Goldenhar syndrome. J. Craniofac. Genet. Dev. Biol.,Copenhagen, v.3, p.313-316, 1983.

15 LESSICK, M.; VASA, R.; ISRAEL, J. Severe manifestations of oculo-auriculo-vertebral spectrum in a cocaine-exposed infant. J. Med. Genet., London, v.28, p.803-804, 1991. 